\title{
Tholins and their relevance for astrophysical issues
}

\author{
E. Quirico ${ }^{1}$, C. Szopa ${ }^{2}$, G. Cernogora ${ }^{2}$, V. Lees ${ }^{3}$, S. Derenne ${ }^{4}$,

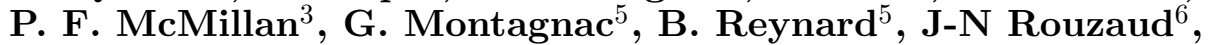

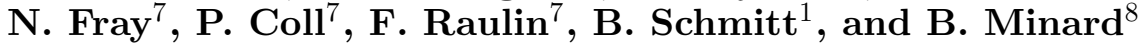 \\ ${ }^{1}$ Laboratoire de Planetologie de Grenoble, University Joseph Fourier CNRS, \\ BP 5338041 Grenoble Cedex 9, France \\ email: eric.quirico@obs.ujf-grenoble.fr \\ ${ }^{2}$ Service d'Aeronomie, Universite Pierre et Marie Curie-Paris 6 et Universite de Versailles \\ Reduit de Verrieres, Route des Gatines, 91371 Verrieres le Buisson, France \\ ${ }^{3}$ Dept. of Chemistry and Materials Chemistry Centre, University College London, \\ 20 Gordon Street, London WC1H 0AJ Great Britain \\ ${ }^{4}$ Laboratoire de Chimie Bioorganique et Organique Physique BioEMCo, Ecole Nationale \\ Superieure de Chimie de Paris, 11, rue Pierre et Marie Curie 75231 Paris Cedex 05 France \\ ${ }^{5}$ Laboratoire de Sciences de la Terre, Ecole Normale Superieure de Lyon, \\ 46 allee d'Italie 69364 Lyon Cedex 7 France \\ ${ }^{6}$ Laboratoire de Geologie, Ecole Normale Superieure de Paris, \\ 24 rue Lhomond 75231 Paris Cedex 5 France \\ ${ }^{7}$ Laboratoire Interuniversitaire des Systemes Atmospheriques, \\ Universite Paris XII - Paris VII, 61 av. du Gal de Gaulle, 94010 Creteil cedex, France \\ ${ }^{8}$ Chemistry Department, Pennsylvania State University, \\ 104 Chemistry Building University Park, PA 16802 United States of America
}

\begin{abstract}
Tholins are polymeric hydrogenated carbon nitrides formed from $\mathrm{N}_{2}: \mathrm{CH}_{4}$ mixtures exposed to electrical discharges. They are complex disordered solids, and their structural chemistry and formation processes are not yet fully understood. Tholins have been widely adopted as useful analogs of reddish organic solids associated with planetary bodies or in interstellar space (e.g., Titan's aerosols, reddish surfaces of outer objects, interstellar organics, etc.) for fitting astronomical observations. However, there has been little evidence to date that they in fact constitute pertinent model materials, i. e. with chemical structure/composition similar to those presumed to be present in planetary or interstellar organic solids. In this contribution, we first review recent advances made regarding the determination of composition and structure of tholins produced in the laboratory. They point to a high chemical selectivity in the range of functional groups present, the control of unsaturation by nitrogen, and the highly disordered character of the structures. In a second section, we discuss the relationship between chemistry and the optical properties of tholins, and we point out the lack of a unique relationship between the shape and strength of the visible absorption bands and the chemical composition or structure of the model tholins. The tholins exhibit similarities with HCN "polymers", that are suspected to be present in cometary refractory dust. This points to the existence of possible similar polymerisation processes, and it suggests they could also be used as analogs of N-rich cometary organics. Laboratory-based studies of cometary dust might offer new insights on the "chemical relevancy" of tholins, as combined micro-analytical techniques will allow direct comparison of chemical information between the materials produced. In a third section we present recent results pertaining to the search for such compounds in cometary grains (Stardust grains, interplanetary dust particles - IDPs). We show that some N-rich spots in stratospheric IDPs are rich in cyanide species, but no tholin-like compounds or polymeric HCN have been detected to date.
\end{abstract}


Keywords. Comets: general, tholins, organic matter, chondrites

\section{Introduction}

Titan's tholins (termed simply tholins in this contribution) are hydrogenated carbon nitrides, synthetized from $\mathrm{N}_{2}: \mathrm{CH}_{4}$ gas mixtures in cold plasma conditions. Many experiments have been carried out worldwide for more than 30 years, producing tholins with various chemical compositions and optical properties. These experiments have been complemented by chemical and physical characterization of the tholins, and systematic investigations have been carried out to understand the control of experimental parameters (Coll et al. 1998, Mutsukura \& Akita 1999, Imanaka et al. 2004, and reviews and references therein).

Tholins have been proposed and are currently used as analogs of Titan's aerosols, reddish surfaces of outer satellites and TransNeptunian Objects, or interstellar organics (Khare et al. 1984, Cruikshank et al. 2005, de Bergh et al. 2008). However, though the properties of some tholins match the optical constants of Titan's aerosols as well as other planetary organics, there is no evidence that their chemical structure is in fact similar. Indeed, one of the major issues today lies in the understanding of the chemical structure of tholins and its relationship to their optical properties. As these materials are mostly insoluble in current solvents, their characterisation calls for a wide range of solid state chemistry approaches.

Several complementary spectroscopic, diffraction and analytic techniques were applied to series of tholins formed using different experimental strategies (Ferrari et al. 2003, Bernard et al. 2006, Derenne et al. 2008, Quirico et al., submitted). The soluble fraction of tholins, which account for much less than $1 \%$ of the total mass, has been analysed by very high resolution mass spectrometry (Sarker et al. 2003). We review the results of these studies in a first section. In a second question, we discuss the relevancy of tholins for simulating planetary organics, and we show that no obvious conclusions can be made from spectral observations made in the visible range. In a third section, we discuss the use of tholins as analogs of N-rich refractory organics present in cometary grains. We explain why they should be used along with the so-called "HCN polymers", and describe the microspectroscopic techniques suitable to identify them in cometary grains.

\section{Composition and structure of Tholins}

From the solid state point of view, tholins constitute disordered or quasi-amorphous solids. Their X-Ray diffraction patterns exhibit a broad continuum, onto which are superimposed occasional weak features indicating the presence of some more highly ordered domains (Figure 1, Quirico et al., submitted). Analysis by High Resolution Transmission Electron Microscopy studies provide similar results, and they rule out the presence of large polycyclic aromatic units.

Infrared spectroscopy reveals that tholins produced using a range of different experimental strategies form a fairly homogeneous family of "polymeric" hydrogenated carbon nitrides. Systematic experiments reveal there is a correlation between the $\mathrm{N}$ abundance and that of aliphatic $\mathrm{CH}_{2} / \mathrm{CH}_{3}$, as well as the absorption in the visible range (Imanaka et al. 2004). Functional groups identified by IR spectroscopy include cyanides and conjugated cyanides (-CN), aliphatic $\mathrm{CH}_{2}$ and $\mathrm{CH}_{3}$, primary amines $\mathrm{NH}_{2}$, and $\mathrm{C}=\mathrm{N}$ bonded 


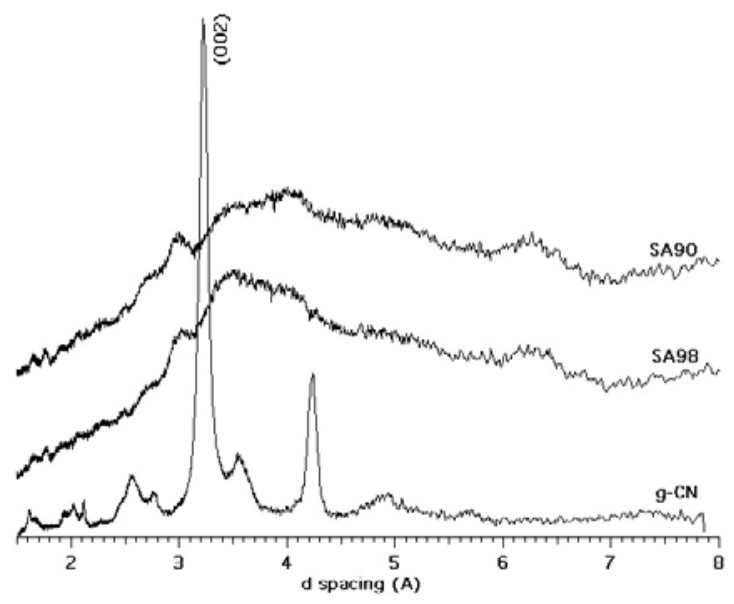

Figure 1. X-ray diffraction (XRD) spectra of two tholins with different elemental compositions compared with a crystalline graphitic carbon nitride produced by high pressure-high temperature synthesis from melamine and cyanuric chloride precursors (Zhang et al. 2004). The tholins exhibit broad diffraction features consistent with a poorly-organised solid.

groups. There is no evidence for any protonated aromatic species, and branched aromatics can not be recognised in such complex spectra (Quirico et al., submitted).

Unlike IR, Raman spectroscopy is particularly sensitive to unsaturated compounds, in particular aromatic species. The use of UV excitation permits spectra to be taken avoiding the strong fluorescence encountered with visible excitation, and leads to spectra exhibiting the first-order carbon bands, as well as features of minor resonantly excited species assigned to - $\mathrm{CN}$ and highly branched $\mathrm{C}_{3} \mathrm{~N}_{3}$ rings (Bernard et al. 2006, Quirico et al., submitted). The abundance of $\mathrm{C}_{3} \mathrm{~N}_{3}$ rings correlates with the $\mathrm{N} / \mathrm{C}$ ratio, consistent with the fact that $\mathrm{N}$ promotes unsaturation. Thus $\mathrm{C}_{3} \mathrm{~N}_{3}$ units appear to constitute an important building block for tholin structures, and such triazine rings could even become dominant in N-rich tholins. The first-order carbon bands are similar to those generally observed in carbon nitrides, and point to very disordered compounds with clustered $s p^{2}$ carbons (in opposition to $\mathrm{C}=\mathrm{C}$ or $\mathrm{C}=\mathrm{N}$ in olefinic chains).

${ }^{13} \mathrm{C}$ and ${ }^{15} \mathrm{~N}$ Nuclear Magnetic Resonance (NMR) has recently bring major clues on tholins composition (Derenne et al. 2008). Thanks to ${ }^{13} \mathrm{C}$ - and ${ }^{15} \mathrm{~N}$-enriched tholins were produced thanks to the PAMPRE experiment located at Service d'Aronomie (Verrieres le Buisson), single pulse and CP-MAS spectra with a high signal to noise ratio could be obtained (Figure 2). Preliminary analysis of these spectra demonstrate the lack of unsaturated carbon species, and in particular the lack of any aromatic species present within the tholins. Triazine rings $\mathrm{C}_{3} \mathrm{~N}_{3}$ and/or imines are suspected from the NMR data, corroborating the UV Raman data, whereas the presence of other N-heteroaromatic rings is unlikely. Aliphatics, cyanides, and amines are definitely detected as major functional groups, consistent with the IR and Raman measurements (Table 1). Further studies should quantify the abundance of these different functional groups.

Fourier transform ion cyclotron resonance (FT-ICR) analysis of soluble molecules in $\mathrm{CH}_{3} \mathrm{OH}: \mathrm{CH}_{3} \mathrm{CN}$ exhibit a wide range of compounds (Sarker et al. 2003). The mass distribution appears very peculiar. Series of molecules are observed as families formed by addition of a $\mathrm{CH}_{2}$ function on the same root-molecule, with a Gaussian mass distribution. For compounds across different series, unsaturation increases both with the $\mathrm{N} / \mathrm{C}$ ratio and the molecular weight. This suggests a continuum between soluble and 


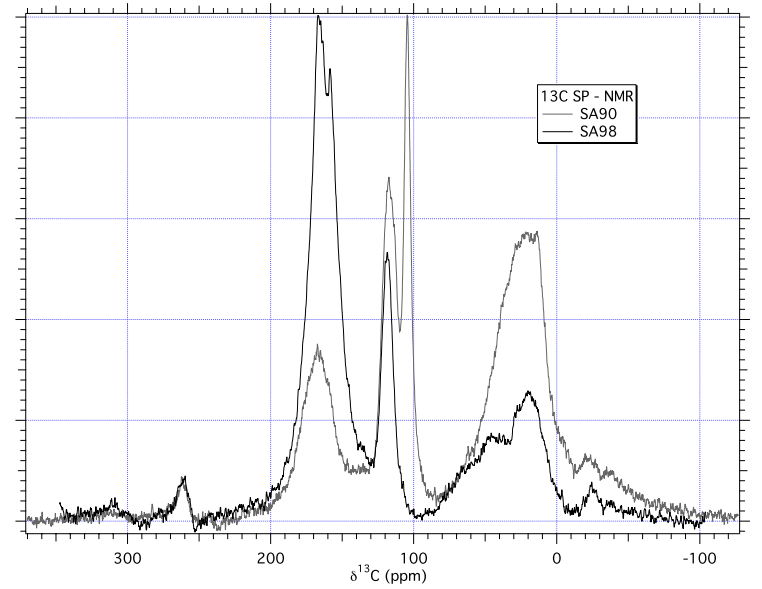

Figure 2. Single pulse ${ }^{13} \mathrm{C}$ NMR spectra of 2 tholins with different elemental compositions. Aliphatic, cyanides, and triazine and/or imines are identified as major compounds in these spectra.

insoluble fractions, and small molecular compounds appear to be of help in understanding the formation process and to identifying the fundamental building blocks of the tholins. Analysis of the soluble fraction appears consistent with the NMR results, as unsaturation is correlated with the presence of nitrogen. The key role of nitrogen in determining the unsaturation index is observed in carbon nitrides films studied in material science, as this atom favors clustering of $s p^{2}$ bondings. FT-ICR results also suggest a random accretion of specific molecular groups around well identified centers, consistent with the chemical selectivity pointed out by NMR and the disordered character of the solid evidenced by Raman spectroscopy and XRD.

\section{Are tholins relevant analogs?}

One of the key question about tholins is their relevancy for simulating extraterretrial organic solids. Because their chemical structure is far from being determined, and that of planetary organics cannot be fully assessed by spectroscopic techniques, no comparison can be achieved. Hence, the relevancy of tholins - as any other analogs like amorphous carbon, soots, etc. - should be defined as their ability to match astronomical data.

In the case of application to understanding Titan's atmosphere, some laboratoryproduced tholins have optical constants in the visible range that are very similar to those of the planetary aerosols (Khare et al. 1984, Imanaka et al. 2004). The physical mechanisms controlling the visible absorption are electronic transitions, between $\pi$ and $\pi^{*}$ states forming valence and conduction bands (Daigo \& Mutsukura 2004). There is however no simple or direct relationship between the electronic structure and the chemical

Table 1. Summary of chemical groups in tholins, as identified, lacking/minor or suspected species.

\begin{tabular}{l|l|l}
\hline Identified & Minor or lacking & Suspected \\
\hline Amines & Isocyanide & Hydrazoles \\
Cyanide & Unsaturated carbons & \\
$\mathrm{CH}_{2} / \mathrm{CH}_{3}$ & Other azines & \\
Triazine, highly branched & Azoles & \\
Imines & Carbodiimide & \\
\hline
\end{tabular}


composition/structure. In other words, different compounds may exhibit similar absorption properties. This has been demonstrated by Tran et al. (2003), who show that various photolysis products exhibit very similar optical constants to those of the tholins of Khare et al. (1984), although they have very different chemical structures and compositions. The tholins are best considered as a class of synthetic hydrogenated carbon nitrides, which should be used in astrophysics like other analogs like amorphous carbon, hydrogenated amorphous carbons, soots, etc. (see de Bergh et al. 2008 for a review), but without any observational evidence they are "chemically pertinent".

Interestingly, the study of cosmomaterials available in the laboratory (e.g., cometary grains) does not suffer from this spectroscopic artifact. Their characterization takes advantage of a large array of micro-analytical techniques, which allow comparison of direct chemical and structural information. The presence of tholins or tholin-like compounds is adressed in the following section in the case of cometary dust.

\section{N-rich organics in cometary dust}

The presence of N-rich refractory organics in cometary grains and Insoluble Organic Matter (IOM) extracted from pristine carbonaceous chondrites has been evidenced by SIMS imagery (Aleon et al. 2003). N abundances up to $20 \mathrm{wt} \%$ have been recorded on tiny areas within these samples $(\sim \mu \mathrm{m})$. HCN polymers have been proposed as analogs of such compounds, regarding their elemental composition and the presence of HCN in cometary ices. Studies of tholins as presented in the first section have also focused on HCN polymers. Both families of compounds exhibit structural and spectroscopic similarities, though they are not identical. This suggests that tholins may also be used as analogs, along with HCN polymers, for IOM and other N-rich refractory organics. Tholins are generally chemically and optically more homogeneous at the micrometric scale, they can be produced under reproducible conditions, and they are likely more stable with time than HCN polymers. Furthermore, depending upon experimental compositions, they can exhibit a much broader range of chemical variations, hence providing a large set of analogs and calibration standards.

There is to date no direct evidence of the presence of HCN polymers or similar carbon nitrides in cometary grains. The characterization of cometary grains or IOMs at the micrometric scale can be achieved by Raman and IR microspectroscopies. $244 \mathrm{~nm} \mathrm{UV}$ Raman micro-spectroscopy, which was used for studying tholins, has been applied to 2 stratospheric IDPs and a series of IOMs extracted from various carbonaceous chondrites (Murchison, Cold Bokkeveld, Murray, Orgueil, Alais, Tagish Lake, Renazzo). The firstorder carbon bands in spectra of IOMs look quite similar, with a narrow and intense G band and a weak D band. For all chondrites studied, no HCN polymers or similar compounds were detected. In the Alais chondrites, the cyanide function has been identified thanks to a very faint band at $2225 \mathrm{~cm}^{-1}$ (Figure 3 ).

The Raman spectra of IDPs were acquired on whole particles without prior demineralization. The most C-rich IDP (L2008X3) exhibited Raman spectra with a useful signal to noise ratio, which all contained the first-order carbon bands at each measurement points (Figures 3 and 4). The shape of these bands are different from either chondritic IOMs or HCN polymers and tholins. They do not appear as a broad feature pointing to an amorphous structure, but rather as two distinct $\mathrm{G}$ and $\mathrm{D}$ bands consistent with a polyaromatic structure, the former being broader than in IOMs and the second more intense. These measurements are consistent with systematic trends observed in $514 \mathrm{~nm}$ Raman spectra (Figure 5), acquired on series of IDPs and carbonaceous chondrites (Quirico et al. 2005, Quirico et al., in preparation). There are several ways for interpreting such data and 


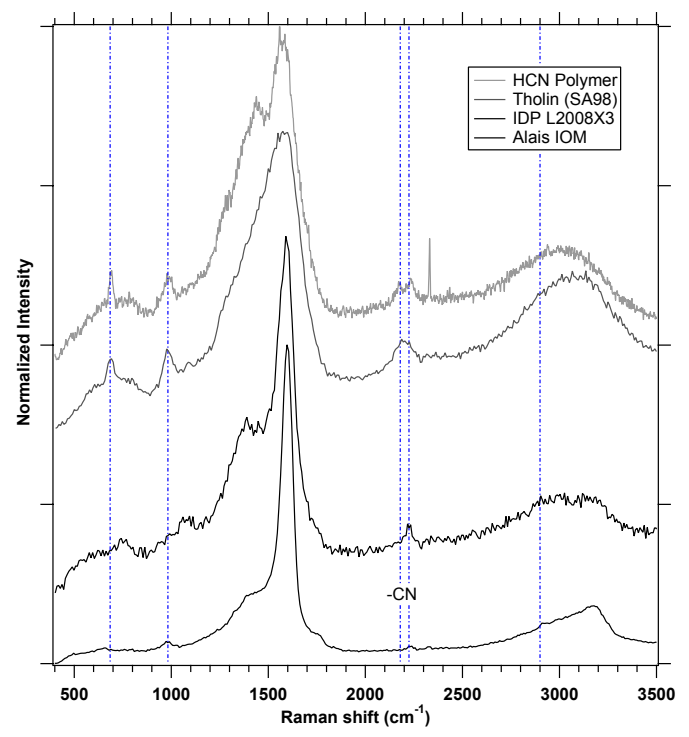

Figure 3. $244 \mathrm{~nm}$ Raman spectra of Alais (CI1) IOM and a HCN polymer.

complementary measurements are required to go further. Note that similar differences have been recently reported between carbonaceous chondrites and Antarctic micro meteorites (AMMs) (Dobrica et al. 2008).

Another significant difference with chondritic IOMs is the presence of spots exhibiting a strong -CN band $\left(2225 \mathrm{~cm}^{-1}\right.$ ) (Figure 3). Such an observation is obviously consistent with the N-rich areas evidenced by SIMS imaging. These results demonstrate that $244 \mathrm{~nm}$ UV Raman spectroscopy is a powerful imaging technique for localizing N-rich organics, and systematic surveys on series of IDPs and AMMs are now in progress. Some N-rich areas are definitely not HCN polymers. More extensive surveys on chondritic IOMs, stratospheric IDPs, and AMMs, combined with SIMS imagery, are under way and these should provide new insights on the presence of tholin-like or HCN polymers.

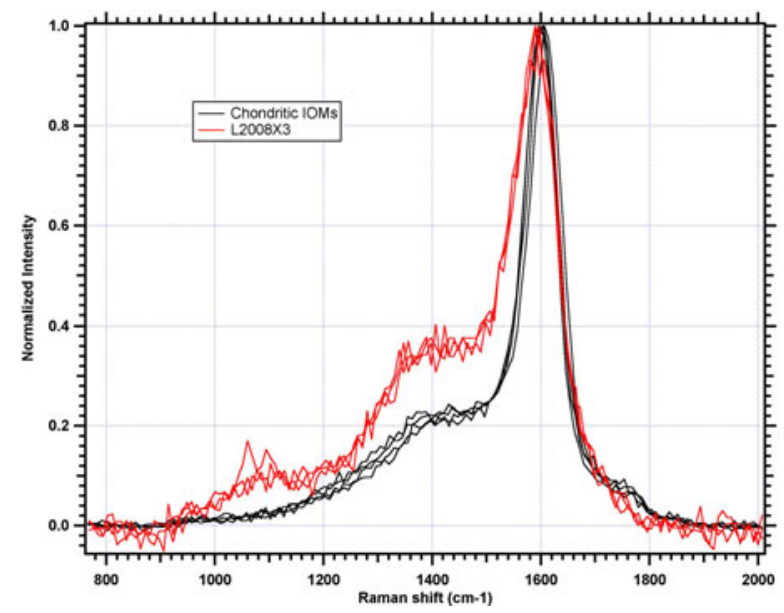

Figure 4. $244 \mathrm{~nm}$ Raman spectra of stratospheric IDP L2008X3 and chondritic IOMs. Note the broader $\mathrm{G}$ band and the more intense $\mathrm{D}$ band. 


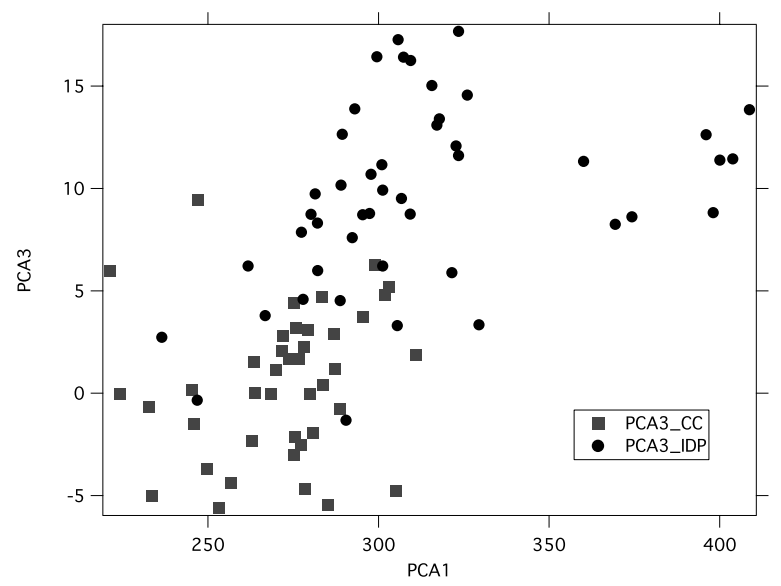

Figure 5. PCA analysis of $514 \mathrm{~nm}$ Raman spectra presented in Quirico et al. (2005). Carbonaceous chondrites and IDPs form distinct groups, suggesting structural differences in their polyaromatic networks.

\section{References}

Aleon, J., Robert, F., Chaussidon, M., \& Marty, B. 2003, Geochim. Cosmochim. Acta, 67, 3773 Bernard, J-M, Quirico, E., Brissaud, O., Montagnac, G., Reynard, B., McMillan, P. F., Coll, P., Nguyen, M-J, Raulin, F., \& Schmitt B. 2006, Icarus, 185, 301

Coll, P., Coscia, D., Gazeau, M-C, Guez, L., \& Raulin, F. 1998, Origin of life and evolution of the biosphere, 28, 195-213

Cruikshank, D. P., Imanaka, \& Dalle Ore, C. M., 2005, Advances in Space Research, 36, 178-183

Daigo, Y. \& Mutsukura, N. 2004, Diam. Rel. Mat., 13, 2170-2173

de Bergh, C., Schmitt, B., Moroz, L. V., Quirico, E \& Cruikshank, D. P. 2008, in The Solar System Beyond Neptune, 483-506

Derenne, S., Quirico, E., Szopa, C., Cernogora, G., Schmitt, B., Lees, V., \& McMillan P. F. 2008, LPSC Meeting, Abstract 1391

Dobrica, E., Engrand, C., Quirico, Montagnac, G., \& Duprat J. 2008, Meteor. and Plan. Sci., Meteoritical Society Meeting, Matsue, Japan, Abstract 50202

Ferrari, A. C., Rodil, S. E., \& Robertson, J. 2003, Phys. Rev. B, 67, 155306

Imanaka, H., Khare, B. N., Elsila, J. E., Bakes, E. L. O., McKay, C. P., Cruikshank, D. P., Sugita, S., Matsui, T., \& Zare, R. N. 2004, Icarus, 168, 344

Khare, B. N., Sagan, C., Thompson, W. R., Arakawa, E. T., Suits, F., Callcott, T. A., Williams, M. W., Shrader, S., Ogino, H., Willingham, M. W., \& Nagy, B. 1984, Adv. Space Res., 4, 59

Mutsukura, N. \& Akita, K-I. 1999, Thin Solid Films, 349, 115-119

Quirico, E., Borg, J., Raynal, P-I, Montagnac, G., \& d'Hendecourt, L. 2005, Plan. Spac. Sci., $53,1443-1448$

Quirico, E., Montagnac, G., Lees, V., McMillan, P. F., Szopa, C., Cernogora, G., Rouzaud, J-N, Simon, P., Bernard, J-M, Coll, P., Fray, N., Minard, R. D., Raulin, F., Reynard, B., \& Schmitt, B., Icarus, submitted

Sarker, N., Somogyi, A., Lunine, J., \& Smith M. A. 2003, Astrobiology, 3, 719

Tran, B. N., Joseph, J. C., Ferris, J. P., Persans, P. D., \& Chera, J. J. 2003, Icarus, 165, 379-390

Zhang, Z., Leinenweber, K., Bauer, M., Garvie, L. A., McMillan, P. F., \& Wolf, G. H. 2004, J. Am. Chem. Soc., 123, 7788-7796

\section{Discussion}

MATTHEws: You have made a direct comparison between a sample of our HCN polymer and various tholins. What are your conclusions about the existence of HCN polymer in the tholins? 
QUIRICO: They are different compounds, but there are some striking similarities. I refer back to the NMR results, which point to the presence of similar functional groups. The main similarity lies in a peak that we assign as imines and/or triazine functional groups, the presence of cyanide, and the lack of any unsaturated carbons (aromatics, etc.). Nevertheless, in both NMR and infrared data, many bands are narrower. Overall, I would say that though there are some similarities in the chemical composition, HCN polymer are chemically simpler than tholins.

ZINNER: I want to make a comment to remove some confusion you might have caused by referring to areas with high nitrogen concentrations as 'hot spots.' The 'hot spots' discussed yesterday in various isotopic talks were regions which have large isotopic anomalies - specifically for nitrogen these are regions with high ${ }^{15} \mathrm{~N}$ excesses, but not an enhancement in the nitrogen concentration.

QuIRICO: The main point I want to make is that there are some areas in the stratospheric dust that show very localized nitrogen concentrations.

NitTler: You did some component analysis and showed a plot that discriminates between the IDPs and the chondrites, but you didn't discuss what the actually components are. Is there any physical reality to the components the analysis gives you?

QuIRICO: They do not have any physical reality. This just provides a means to characterize the spectral variation, which is more sensitive than current fits using two bands.

Cody: UV Raman spectroscopy gives you enormous signal enhancement, but it can also cause some photochemistry. Are you concerned about photochemical alteration caused by exposure of the IDP organics or the meteoritic organics to your UV laser?

QUIRICO: The issue of photostability is a major issue in Raman spectroscopy. There are four major problems, whatever the wavelength excitation: heating (reversible); annealing; photooxidation and photolysis. We used considerable care to minimize those effects, by using a rotating sample holder, and performing time-resolved measurements at the same spot location. Though we cannot exclude the full lack of sample alteration, those effects were strongly minimized in our experiences. 